\title{
Carnitine acetyltransferases are required for growth on non-fermentable carbon sources but not for pathogenesis in Candida albicans
}

\author{
Huaijin Zhou and Michael C. Lorenz
}

Correspondence

Michael C. Lorenz

Michael.Lorenz@uth.tmc.edu

Received 1 November 2007

Revised 13 November 2007

Accepted 14 November 2007

\author{
Department of Microbiology and Molecular Genetics, The University of Texas Health Science \\ Center at Houston, 6431 Fannin Street, Houston TX 77030, USA
}

\begin{abstract}
Carbon starvation is a significant stress encountered by the opportunistic fungal pathogen Candida albicans, and mutations in several pathways required to assimilate non-fermentable carbon sources attenuate virulence. These pathways - $\beta$-oxidation, the glyoxylate cycle and gluconeogenesis - are compartmentalized in the fungal cell between the peroxisome, mitochondria and cytosol; thus, the cell must transport key intermediates between these organelles. Transport of acetyl-CoA, a particularly important intermediate of carbon metabolism, is catalysed by membrane-associated carnitine acetyltransferases (CATs). We report here the characterization of the three predicted CAT genes in C. albicans, CTN1, CTN2 and CTN3. Strains lacking CTN1 or CTN2 were unable to grow on ethanol or acetate as sole carbon source; additionally, citrate was utilized poorly $(\Delta c t n 2)$ or not at all $(\Delta c t n 1)$ and the $\Delta c t n 2$ mutant failed to grow on fatty acids as well. In contrast, deletion of CTN3 had no observable phenotype. All three genes were upregulated in the presence of non-fermentable carbon sources and after macrophage phagocytosis. CTN1 and CTN3 were able to complement the corresponding Saccharomyces cerevisiae $\Delta y a t 1$ and $\Delta y a t 2$ mutants. However, these mutants had no obvious attenuation in virulence in a mouse model of disseminated candidiasis, in contrast to other carbon metabolism mutants. These findings extend our understanding of nutrient stress in vivo and in vitro and the contribution of metabolic pathways to virulence in C. albicans.
\end{abstract}

\section{INTRODUCTION}

The polymorphic yeast Candida albicans is the most common fungal pathogen of humans and it can infect virtually any tissue and organ within the body. The most serious manifestation, disseminated haematogenous candidiasis, has a mortality rate of $\sim 40 \%$ (Wenzel, 1995; Wisplinghoff et al., 2004). C. albicans is also, however, a ubiquitous and harmless part of our normal microbiota. The transition from commensal to pathogen generally results from changes in the host innate immune system. Therefore, the interaction between this fungal pathogen and phagocytic cells is a key to disease development.

We focus on the interaction between macrophages and $C$. albicans. In vitro, C. albicans is able to survive phagocytosis by differentiating into elongated hyphae that eventually disrupt the macrophage. An extensive microarray analysis of the Candida-macrophage interaction revealed a very complex programme of gene expression in response to phagocytosis in this fungus; a substantial part of this programme was the upregulation of specific metabolic pathways and stress response systems (Lorenz et al., 2004).

Abbreviation: CAT, carnitine acetyltransferase.
Expression analysis of neutrophil-C. albicans interactions revealed a similarly complex response, but one that was quite different in terms of the pathways and processes induced (Fradin et al., 2003; Rubin-Bejerano et al., 2003). Thus it is clear that $C$. albicans elaborates a very sophisticated response to immune cell contact.

The transcript profiles of phagocytosed cells resemble those of cells starved for carbon, with particular induction of pathways to utilize alternative or non-fermentable carbon sources (Lorenz et al., 2004). Like other fungi, C. albicans uses glucose as the preferred carbon source, but can utilize a variety of non-fermentable carbon sources such as ethanol, acetate, citrate, lactate and oleate. These alternative carbon sources are assimilated through three main metabolic pathways: $\beta$-oxidation, the glyoxylate cycle and gluconeogenesis. We and others have shown that disabling these pathways through the mutation of key enzymes causes varying degrees of attenuation of virulence in a mouse model of disease (Barelle et al., 2006; Lorenz \& Fink, 2001; Piekarska et al., 2006; Ramírez \& Lorenz, 2007). These studies make it clear that carbon starvation is a relevant and important stress encountered by C. albicans during infection; as a result we are interested in further defining metabolic requirements in vivo. 
A key intermediate in alternative carbon metabolism is acetyl-CoA, which is produced by the breakdown of ethanol, fatty acids and some amino acids, and is used by the glyoxylate and TCA cycles. Some of these processes are compartmentalized in the fungal cell, occurring in the peroxisome, mitochondria or cytosol, necessitating intracellular transport of acetyl-CoA. This is facilitated by carnitine acetyltransferases (CATs) that catalyse the transfer of the acetyl group from acetyl-CoA to carnitine followed by the transport of acetylcarnitine across the membrane. This process is referred to as the 'carnitine shuttle' (Swiegers et al., 2001) and is important during growth on non-fermentable carbon sources. Saccharomyces cerevisiae has three CAT genes, YAT1, YAT2 and CAT2 (Swiegers et al., 2001). C. albicans also has three predicted CAT genes, CTN1, CTN2 and CTN3 (Prigneau et al., 2004); these genes are significantly upregulated by macrophage phagocytosis (Lorenz et al., 2004; Prigneau et al., 2004).

Here we describe the characterization of the CTN genes in C. albicans. To this end, we generated deletion mutants in the CTN1, CTN2 and CTN3 genes and tested their growth in vitro and in vivo. $\Delta c t n 1$ and $\Delta c t n 2$ mutants had an overlapping spectrum of growth defects in the presence of alternative carbon sources: the $\Delta c t n 1$ strain could not utilize acetate, ethanol or citrate while the $\Delta c t n 2$ mutant did not grow on acetate, ethanol or oleate and grew poorly on citrate. The $\Delta c t n 3$ mutant had no obvious phenotype, as previously reported (Prigneau et al., 2004). These phenotypes are more severe than the $S$. cerevisiae CAT mutants, reinforcing recent findings that there are significant differences in carbon metabolism between the two species. We verified that all three genes were induced in the macrophage, as well as on some non-fermentable carbon sources. We also showed that C. albicans CTN1 and CTN3 complement the corresponding S. cerevisiae mutants. None of these mutants, however, affected virulence in a mouse model of disseminated candidiasis. These findings extend our understanding of alternative carbon source utilization pathways in C. albicans and their role in pathogenesis.

\section{METHODS}

Strain construction. Strains are listed in Table 1. The C. albicans $\Delta c t n 1, \Delta c t n 2$ and $\Delta c t n 3$ mutants were constructed using the SAT1flipper cassette (Reuß et al., 2004). Briefly, 600 bp fragments flanking the gene of interest were cloned between KpnI and XhoI ( $\left.5^{\prime}\right)$ or SacII and SacI $\left(3^{\prime}\right)$ sites in pSFS1. The resulting plasmid was linearized with $K p n I$ and SacI and the disruption cassette was used to transform strain SC5314 by electroporation (Reuß et al., 2004) with selection on YPD plates containing $100 \mu \mathrm{g}$ nourseothricin $\mathrm{ml}^{-1}$. Correct integration was confirmed by $\mathrm{PCR}$, and excision of the Nour ${ }^{\mathrm{R}}$ cassette was induced by growth in YCB-BSA at $30{ }^{\circ} \mathrm{C}$ for $24 \mathrm{~h}$. Proper excision was confirmed by PCR. The second allele was deleted in the same manner. Mutants were complemented by reintroducing the ORF at its native locus. The upstream fragment of the disruption construct was replaced with the complete ORF; the transforming and selecting were the same as for the disruptions. The double mutant $\Delta c t n 1 \Delta c t n 3$ was constructed by sequentially deleting the two genes.

The S. cerevisiae mutants $\Delta y a t 1, \Delta y a t 2, \Delta$ cat2 and $\Delta$ cit2 were obtained from the haploid collection made by the Saccharomyces Genome

Table 1. Strains

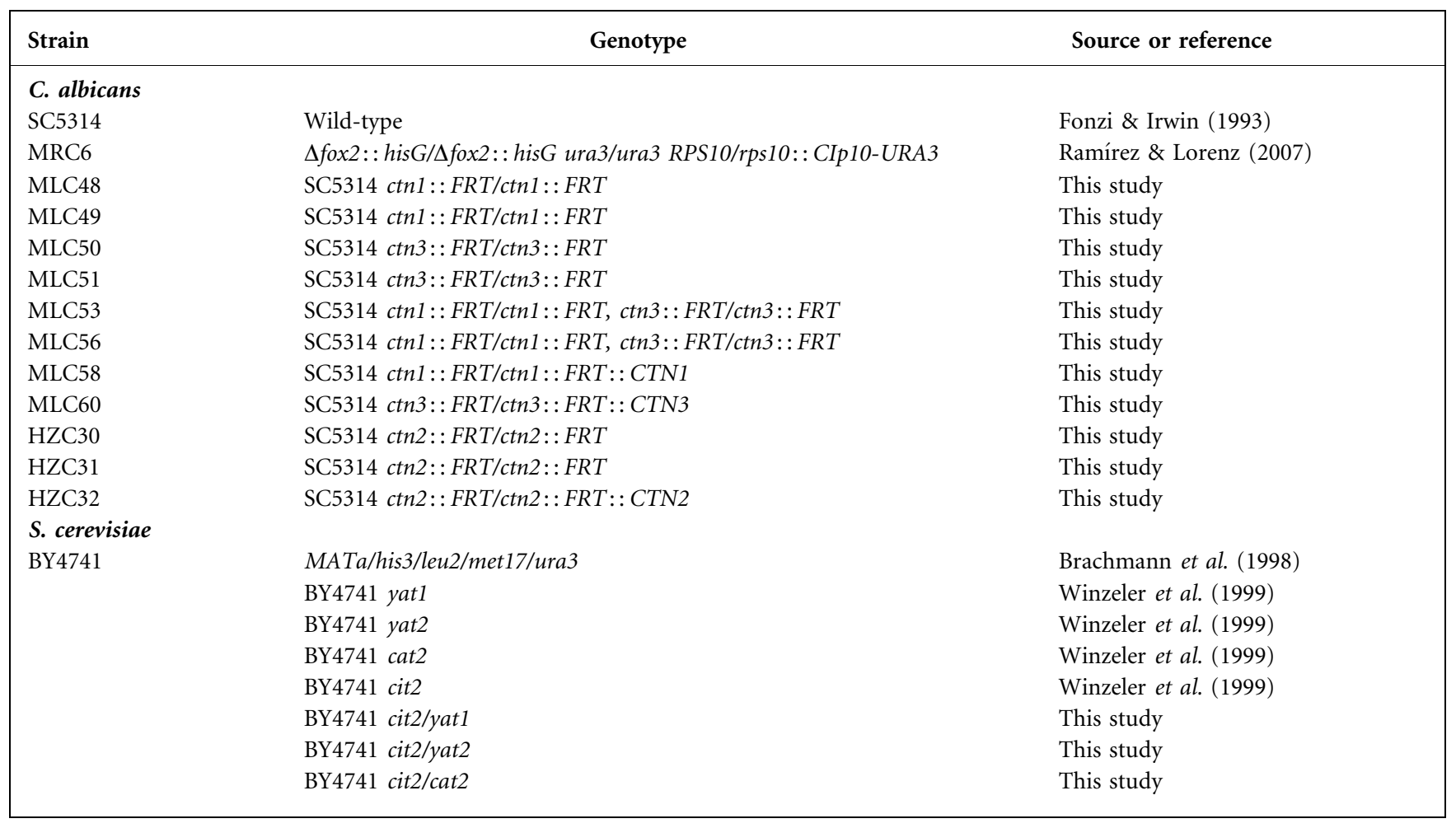


Deletion Project (Winzeler et al., 1999). $\Delta y a t 1 \Delta$ cit2, $\Delta y a t 2 \Delta$ cit2 and $\Delta$ cat2 $\Delta$ cit2 double mutants were constructed by introducing $\Delta$ cit2::Nour ${ }^{R}$ into $\Delta y a t 1, \Delta y a t 2$ and $\Delta$ cat2 strains using a PCR disruption approach (Wach et al., 1994) using the Nour resistance cassette from pAG25 (Goldstein \& McCusker, 1999). Correct double mutants were confirmed by PCR.

Plasmid construction. To make $S$. cerevisiae overexpression plasmids, the ScYAT1, ScYAT2 and ScCAT2 ORFs were PCR amplified and cloned between EcoRI and HindIII sites in pRS426GPD, a high-copy vector with the constitutive GPD1 promoter (Mumberg et al., 1995); CaCTN1 and CaCTN3 were cloned between BamHI and Sall sites; CaCTN2 was cloned between BamHI and XhoI sites. Plasmids used in this study are listed in Table 2.

In vitro growth assays. To test the growth of C. albicans strains on different carbon sources, the strains were grown in YPD to midexponential phase. Cells were collected by centrifugation and washed with water. They were transferred to a 96 -well plate at $\mathrm{OD}_{600} \sim 0.5$ and serially diluted fivefold. They were spotted using a multiprong pin replicator onto solid YNB medium containing $2 \%$ glucose, potassium acetate, ethanol, sodium citrate, lactate or oleate as the sole carbon source, and incubated at $30{ }^{\circ} \mathrm{C}$ for $2-7$ days, depending on the carbon source.

For the $S$. cerevisiae complementation assays, mutant strains $\Delta y a t 1$ $\Delta$ cit $2, \Delta$ yat $2 \Delta$ cit 2 and $\Delta$ cat $2 \Delta$ cit 2 were transformed with the pRS426GPD plasmids. Transformants were patched to YNB medium containing $2 \%$ glucose and amino acids to satisfy growth requirements. Plates were incubated at $30{ }^{\circ} \mathrm{C}$ for 3 days and replica plated to media containing $2 \%$ glucose, or $2 \%$ ethanol plus $10 \mu \mathrm{g}$ carnitine $\mathrm{ml}^{-1}$, and incubated at $30{ }^{\circ} \mathrm{C}$ for 3-10 days.

Northern analysis. SC5314 cells were grown in YNB with $2 \%$ glucose overnight, collected by centrifugation, washed twice with water, resuspended in YNB with $2 \%$ glucose, acetate, oleate or lactate and grown for $1 \mathrm{~h}$. Cells were collected by centrifugation and frozen in dry ice-ethanol. Total RNA was extracted using the hot acid phenol method as described by Ausubel et al. (1993). Fifteen nanograms of each sample was separated on a $1 \%$ MOPS-agarose gel with formaldehyde and then transferred to a nylon membrane. Genespecific probes were amplified by PCR and were labelled with $\left[{ }^{32} \mathrm{P}\right] \mathrm{dCTP}$ using the RadPrime DNA labelling system (Invitrogen). Probes were purified using Roche Quick Spin columns. Blots were incubated in pre-hybridization solution containing $5 \times$ SSC $(1 \times$ SSC is $0.15 \mathrm{M} \mathrm{NaCl}, 0.015 \mathrm{M}$ sodium citrate), $50 \%$ formamide, $5 \times$ Denhardt's solution, $0.1 \%$ SDS and $100 \mu \mathrm{g} \mathrm{ml}^{-1}$ single-stranded DNA for $2 \mathrm{~h}$ at $42{ }^{\circ} \mathrm{C}$ followed by hybridization overnight. rRNA was used as a loading control.
RT-PCR. Cells $\left(4 \times 10^{7}\right)$ of the murine macrophage line J774A were plated in a $750 \mathrm{ml}$ tissue culture flask in RPMI with $10 \%$ FBS and incubated overnight at $37{ }^{\circ} \mathrm{C}$ with $5 \% \mathrm{CO}_{2}$. Overnight cultures of strain SC5314 were diluted 1:50 in YPD and grown for $\sim 4 \mathrm{~h}$ at $30{ }^{\circ} \mathrm{C}$. Cultures were collected by centrifugation, washed in PBS and counted using a haemocytometer; $10^{8}$ C. albicans cells were added to the flasks containing macrophages (to give a $C$. albicans : macrophage ratio of approx. 2:1) and incubated for $1 \mathrm{~h}$. For control cultures, $2 \times 10^{8} \mathrm{C}$. albicans cells were inoculated to tissue culture flasks in $50 \mathrm{ml}$ RPMI plus $10 \%$ serum. Cells were collected by scraping the flasks with rubber scrapers in ice-cold water and transferring to $50 \mathrm{ml}$ conical tubes. Cells were centrifuged at 3000 r.p.m. at $4{ }^{\circ} \mathrm{C}$, washed twice with ice-cold water, transferred to microfuge tubes, and frozen on dry ice. Total RNA was isolated by hot phenol extraction (Ausubel et al., 1993). First-strand cDNA was synthesized according to the Invitrogen SuperScriptII RT protocol. The cDNA product was diluted $1: 10$ twice and $2 \mu \mathrm{l}$ of each dilution was used as template in the PCR reaction. Primers were designed to amplify a $\sim 300 \mathrm{bp}$ internal fragment of CTN1, CTN2 or CTN2, or 18S rRNA as a control.

Filamentation assay. C. albicans strains from fresh YPD plates were

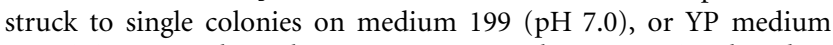
containing $3 \%$ glycerol or $10 \%$ serum. Plates were incubated at $37{ }^{\circ} \mathrm{C}$ for 4 days. Colony morphology was examined under a Zeiss dissecting scope. Images were assembled in Adobe Photoshop.

In vivo virulence assays. Mouse virulence assays were carried out as described previously (Ramírez \& Lorenz, 2007). Adult, female, outbred ICR mice (21-25 g) were obtained from Harlan. Cultures of C. albicans were grown in YPD and serially passaged twice by overnight growth at $30{ }^{\circ} \mathrm{C}$. Cells were collected by centrifugation, washed and resuspended in PBS. Mice were infected through tail vein injections with $10^{6}$ C. albicans yeast-form cells in $100 \mu \mathrm{l}$ PBS. The group size was 10 mice per strain. Infected mice were monitored for signs of infection and euthanized when moribund according to approved protocols. Survival data were analysed with Prism3 (Graphpad Software) using the log rank test. All animal assays were conducted in accordance with protocols approved by the University of Texas Health Science Center Animal Welfare Committee.

\section{RESULTS}

Carbon metabolic pathways are compartmentalized into several organelles in eukaryotes, including the mitochondria and peroxisome, as well as the cytosol, and several 'shuttles' facilitate the intracellular transport of various intermediates. Acetyl-CoA, the most important of these

Table 2. Plasmids

\begin{tabular}{|c|c|c|}
\hline Plasmid & Description & Resource or reference \\
\hline pSFS1 & $F R T / \mathrm{P}_{\mathrm{SAP} 2} F L P / S A T 1 / F R T$ & Reuß et al. (2004) \\
\hline pAG25 & natMX4 & Goldstein \& McCusker (1999) \\
\hline pRS426-GPD & & Mumberg et al. (1995) \\
\hline pHZ110 & pRS426-GPD-YAT1 $1_{S c}$ & This study \\
\hline pHZ111 & pRS426-GPD- $Y A T 2_{S c}$ & This study \\
\hline pHZ112 & pRS426-GPD-CAT2 $2_{S c}$ & This study \\
\hline pHZ113 & pRS426-GPD-CTN1 $\mathrm{Ca}$ & This study \\
\hline pHZ114 & pRS426-GPD-CTN3 $\mathrm{Ca}$ & This study \\
\hline pHZ115 & pRS426-GPD-CTN2 $\mathrm{Ca}$ & This study \\
\hline
\end{tabular}




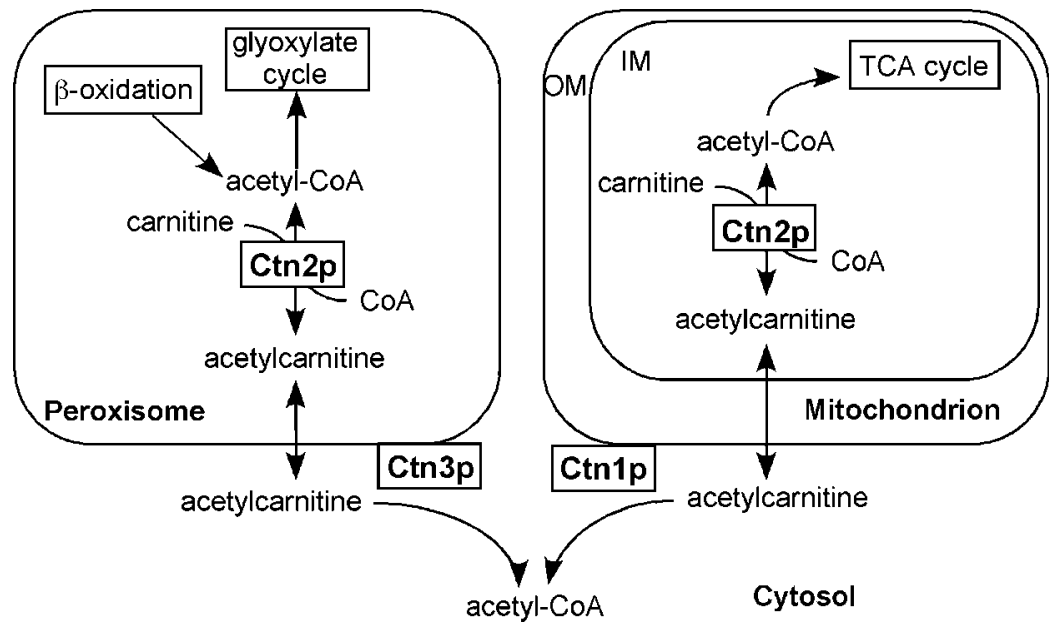

Fig. 1. Schematic representation of the compartmentalization of alternative carbon sources, metabolic pathways and the carnitine shuttle. The C. albicans CATs Ctn1p, Ctn2p and $C \operatorname{tn} 3 p$ are shown with their predicted localization and function. The figure is adapted from Swiegers et al. (2001).

intermediates, is transported by three CATs. Fig. 1 shows the organization of these pathways inside the cell and the 'carnitine shuttle' that serves to transport the key intermediate acetyl-CoA between the compartments.

\section{Mutant strain construction}

There are three genes coding for predicted CATs in $C$. albicans: CTN1 (YAT1, CAT1, orf19.4551), CTN2 (CAT2, orf19.4591) and CTN3 (YAT2, CAT3, orf19.2809). All three are upregulated significantly following macrophage phagocytosis (by 20.4-, 7.2- and 3.9-fold, respectively; Lorenz et al., 2004). Ctn1p is predicted to be associated with the outer mitochondrial membrane and is $58 \%$ similar to $S$. cerevisiae YAT1. Ctn3p has two peroxisome-targeting signal motifs; otherwise it is $53 \%$ similar to the cytosolic ScYat2p. CTN2 has two predicted translation initiation sites, with a mitochondrial targeting signal after the first methionine, and a peroxisome-targeting signal after the second methionine, suggesting a dual localization. This is a similar architecture to the yeast CAT2 gene, whose product is both peroxisomal and mitochondrial (Elgersma et al., 1995). These proteins are $63 \%$ similar to each other (Prigneau et al., 2004).

To disrupt these three genes, we used the SAT1 flipper approach (Reuß et al., 2004) to precisely remove the ORFs of CTN1, CTN2 and CTN3 (see Methods). The SAT1 cassette (encoding resistance to nourseothricin) was excised and the second allele was disrupted in the same way. All three mutants were complemented by inserting one copy of the ORF back at its original locus as described (Reuß et al., 2004; see Methods). We also constructed a $\Delta c t n 1 \Delta c t n 3$ double mutant as part of the process of making a strain lacking all three CTN genes (we have not tested the $\Delta c t n 1 \Delta c t n 2$ or $\Delta c t n 2 \Delta c t n 3$ combinations). To date we have been unable to construct this triple mutant strain, which may indicate that this is an essential gene family. All mutants described here are homozygous deletions unless stated otherwise (see Table 1).

\section{CTN mutants do not grow on certain non- fermentable carbon sources}

We used a spot dilution assay to test the ability of the CAT mutant strains to assimilate different carbon sources. Strains were grown to exponential phase, washed and serially diluted, then spotted using a pin replicator to minimal YNB medium containing $2 \%$ glucose, potassium acetate, ethanol, sodium citrate, lactate or the monounsaturated fatty acid oleate as the sole carbon source (Fig. 2). We included a $\Delta$ fox 2 mutant, lacking the $\beta$-oxidation gene, as a control; this mutant has pleiotropic defects on several carbon sources (Ramírez \& Lorenz, 2007; Piekarska et al., 2006).

As shown in Fig. 2, the $\Delta c t n 1$ mutant was unable to grow on ethanol, potassium acetate and sodium citrate, although its growth on oleate and lactate was indistinguishable from the wild-type strain in this plate assay. The $\Delta \operatorname{ctn} 3$ mutant did not show defects in growth on any of the carbon sources tested; this mutant was previously reported to have no phenotype in the presence of glucose, acetate or methanol (Prigneau et al., 2004). The double $\Delta c t n 1 \Delta c t n 3$ mutant had the same phenotype as the single $\Delta$ ctn 1 mutant. The $\Delta c t n 2$ mutant failed to grow on media containing acetate, ethanol, or oleate and was significantly compromised on citrate. Growth was restored in the complemented strains. We also tested a second, independent mutant for each gene and observed identical results (data not shown).

These findings are in contrast to S. cerevisiae, in which the $\Delta y a t 1, \Delta y a t 2$ and $\Delta c a t 2$ mutants do not have any growth defect on non-fermentable carbon sources unless the peroxisomal citrate synthase CIT2 is also mutated (van Roermund et al., 1995; Swiegers et al., 2001). Though these proteins and metabolic pathways are very highly conserved, several similar phenotypic discrepancies have been recently described (Piekarska et al., 2006; Ramírez \& Lorenz 2007; Sexton et al., 2007; M. A. Ramírez \& M. C. Lorenz, unpublished observations), suggesting that there has been 


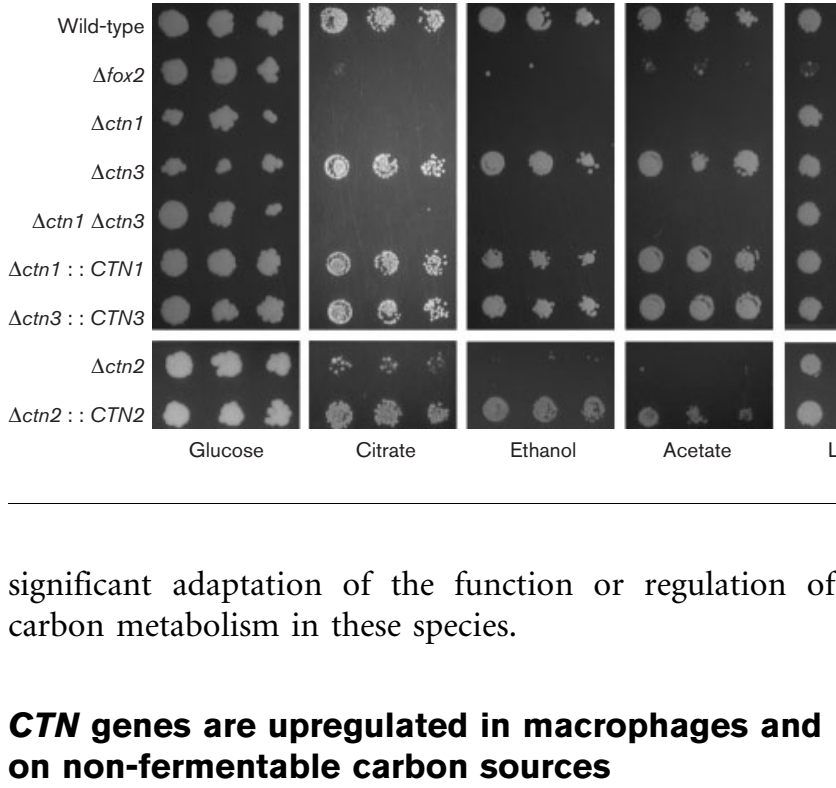

To address the regulation of these genes in C. albicans, we assayed gene expression following phagocytosis and in the presence of various carbon sources. All three CTN genes have been shown to be induced following macrophage phagocytosis by microarray (Lorenz et al., 2004) and Northern analysis (Prigneau et al., 2004). CTN3, additionally, was repressed by glucose and induced by multiple carbon sources including glycerol, oleate, serum, acetate, ethanol and methanol (Prigneau et al., 2004). Given the homology between these genes, there is some concern that cross-hybridization could complicate this analysis, so we re-examined gene expression, testing for specificity using our knockout strains.

We collected wild-type C. albicans (SC5314) $1 \mathrm{~h}$ after macrophage phagocytosis and assayed gene expression by RT-PCR (Fig. 3a). CTN1 and CTN3 were strongly upregulated, as shown previously (Lorenz et al., 2004; Prigneau et al., 2004), though we were unable to demonstrate induction of CTN2 (which was the least induced CAT by microarray). The RT-PCR primers did not amplify a band from the corresponding mutant strains, demonstrating specificity (data not shown).

We next examined the expression pattern of these genes in different carbon sources by Northern blot analysis (Fig. 3b). None of the CAT-encoding genes were detectable in glucoseor lactate-grown cells; CTN1 and CTN3 were induced in acetate-grown cells and further induced moderately (CTN3) or dramatically (CTN1) in oleate. CTN2 was detectable only after growth on the fatty acid oleate (Fig. 3b). The probes are specific to each gene and there was no cross-hybridization (data not shown). In summary, all three CAT genes were upregulated on non-fermentable carbon sources, most strongly in cells exposed to oleate.

The induction of the CAT genes by non-fermentable carbon sources is similar to that of the S. cerevisiae homologues. In S. cerevisiae YAT1 was repressed by glucose and induced by ethanol (Schmalix \& Bandlow, 1993). CAT2 was induced tenfold in oleate as compared to glucose (Atomi et al., 1993).

\section{CTN1 and CTN3 complement S. cerevisiae mutants}

The C. albicans CTN genes are annotated based on their sequence homology to S. cerevisiae YAT1, YAT2 and CAT2. To determine the validity of these assignments, we tested whether the C. albicans $C T N$ genes can complement the $S$. cerevisiae mutant phenotypes. Since in $S$. cerevisiae, mutation in CAT genes alone does not yield a growth defect on non-fermentable carbon sources, we introduced the $\Delta$ cit 2 mutation into $\Delta y a t 1, \Delta y a t 2$ and $\Delta$ cat 2 strains (see Methods). Indeed, as previously described (Swiegers et al., 2001), the double mutants were unable to grow on ethanol as sole carbon source (Fig. 4 and data not shown). The double mutant strains were then transformed with highcopy plasmids containing the CAT genes from $S$. cerevisiae or C. albicans under the control of a constitutive GPD1 promoter.

As shown in Fig. 4, the $\Delta$ cit $2 \Delta$ cat double mutant strains transformed with empty vector were unable to grow on ethanol as the sole carbon source, and overexpression of the $S$. cerevisiae genes complements these phenotypes. $\mathrm{CaCTN} 1$ restores growth on ethanol in both the $\Delta y a t 1$ and $\Delta$ yat2 mutants, while CaCTN3 complements only $\Delta$ yat2. We were unable to complement $\Delta$ cat 2 with any of the $C$. albicans genes, and the CaCTN2 gene did not function in $S$. cerevisiae. This can occasionally be the result of the altered codon usage in C. albicans, but we found no CTG codons in CTN1 or CTN2. CTN3, which is functional in the $S$. cerevisiae $\Delta y a t 2$ mutant, has five CTG codons. Thus, this analysis confirms that there is some conservation of function in this gene family but again suggests that there are some significant differences in carbon metabolism between these related species.

\section{CTN mutants undergo hyphal morphogenesis on solid media}

In a previous study, deletion of CTN3 was found to disrupt filamentation on solid media (Prigneau et al., 2004). We 
(a)

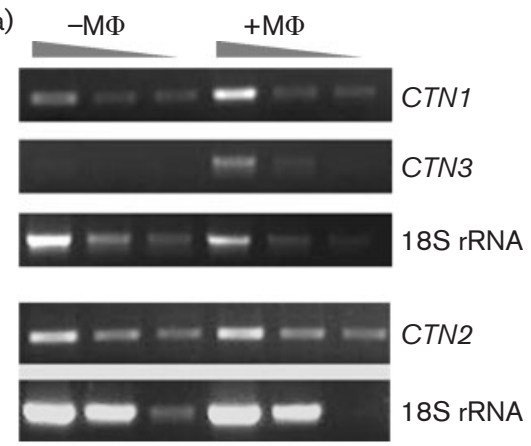

(b) Glu Ace Ole Lac

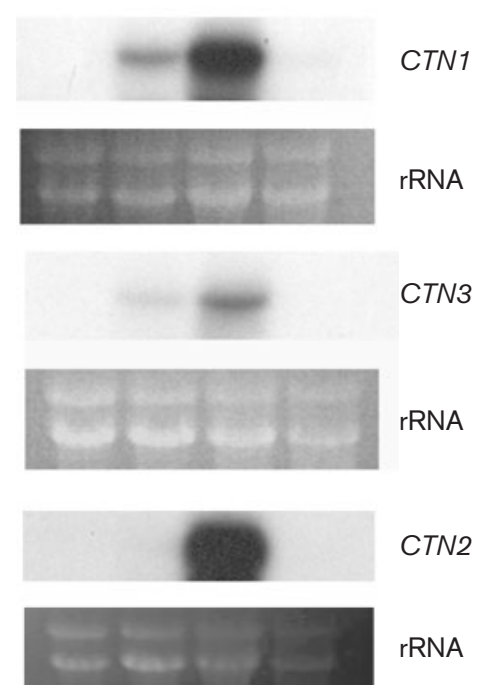

Fig. 3. Expression pattern of CTN1, CTN3 and CTN2. (a) Wildtype (SC5314) cells were added to flasks containing macrophages ( $10^{8}$ cells, giving a $2: 1$ C. albicans: macrophage ratio) in RPMI plus $10 \%$ serum and incubated for $1 \mathrm{~h}$. Control cells were incubated in the same medium without macrophages. Cells were harvested and RNA extracted. The expression of these genes was determined by RT-PCR. For each gene/condition, three $1: 10$ serial dilutions of the RNA (indicated by the gradient sign) were amplified (see Methods). 18S rRNA was amplified as a control. (b) SC5314 was grown to mid-exponential phase in minimal YNB medium with glucose, collected by centrifugation, washed with water, and shifted to medium containing $2 \%$ glucose (Glu), potassium acetate (Ace), oleate (Ole) and lactate (Lac) for $1 \mathrm{~h}$. RNA was extracted from these cells and gene expression was determined by Northern analysis.

tested whether the deletion of the other two genes also affects colony morphology under the same conditions. The wild-type and $\Delta c t n 1, \Delta c t n 2$ and $\Delta c t n 3$ mutant strains were incubated on solid YP-glycerol, YP-serum or medium 199. After 4 days of growth at $37^{\circ} \mathrm{C}$, all four strains formed long peripheral hyphae (Fig. 5 and data not shown), with no difference between the wild-type and the mutant strains under the conditions tested. In contrast to the earlier report for $\Delta c t n 3$, we found that none of these mutants were affected in their filamentation ability. This difference in phenotype may be due to the URA3 deletion marker used in the earlier study; chromosomal position effects of this gene have been found to alter several phenotypes, including hyphal differentiation (Bain et al., 2001; Lay et al., 1998).

\section{CTN mutants are not attenuated in virulence in the mouse model}

Previous work has demonstrated that perturbations in alternative carbon source utilization pathways led to mild to significant attenuation in virulence in a mouse model of haematogenously disseminated candidiasis. These include $\beta$-oxidation of fatty acids via the $\Delta$ fox 2 mutation (Ramírez \& Lorenz, 2007; Piekarska et al., 2006), the glyoxylate cycle via the $\Delta$ icl1 mutation (Ramírez \& Lorenz, 2007; Lorenz \& Fink, 2001), and gluconeogenesis via the $\Delta f b p 1$ (Ramírez \& Lorenz, 2007) or $\Delta p c k 1$ (Barelle et al., 2006) mutations. To determine if CATs play any role in survival and pathogenesis in vivo, we tested the $\Delta c t n$ mutants in a standard mouse tail vein injection model. Briefly, $10^{6} \mathrm{C}$. albicans cells (yeast form) were injected into outbred adult female mice. Infected mice were monitored for signs of infection and euthanized when moribund according to approved protocols.

Mice infected with wild-type strains had a mean time to death (MTD) of 3.9 days (Fig. 6). Mice infected with the mutants had a comparable death rate, with the MTD for $\Delta c t n 1, \Delta t_{n} 2$ and $\Delta c t n 3$ being $4.8,4.0$ and 4.2 days respectively (Fig. 6a, b, c). The double mutant $\Delta c t n 1$ $\Delta c t n 3$ behaved similarly (Fig. 6d). We also tested an independent strain for each mutant and found the same results. Thus, deletions in the CTN genes have no obvious effect on in vivo survival and virulence even though they caused defects in carbon utilization in vitro that were similar to other avirulent mutants.

\section{DISCUSSION}

In this study, we have examined the function and regulation of the three predicted $C$. albicans CAT genes, CTN1, CTN2 and CTN3. Mutations in two of the three genes (CTN1, CTN2) confer growth defects in the presence of non-fermentable carbon sources and all three genes are induced under these conditions. However, strains lacking one or two of these genes retain full virulence, marking a discrepancy between in vitro carbon utilization and in vivo virulence phenotypes, in contrast to other recent reports for metabolic genes (Piekarska et al., 2006; Ramírez \& Lorenz, 2007).

Both the C. albicans $\Delta c t n 1$ and $\Delta c t n 2$ mutant strains were unable to grow on media containing ethanol or acetate. Both of these compounds are converted to acetyl-CoA and transported into the mitochondria via the carnitine shuttle, where they can be assimilated via the TCA and/or glyoxylate cycles. Ctn $2 p$ is predicted to be in both the 


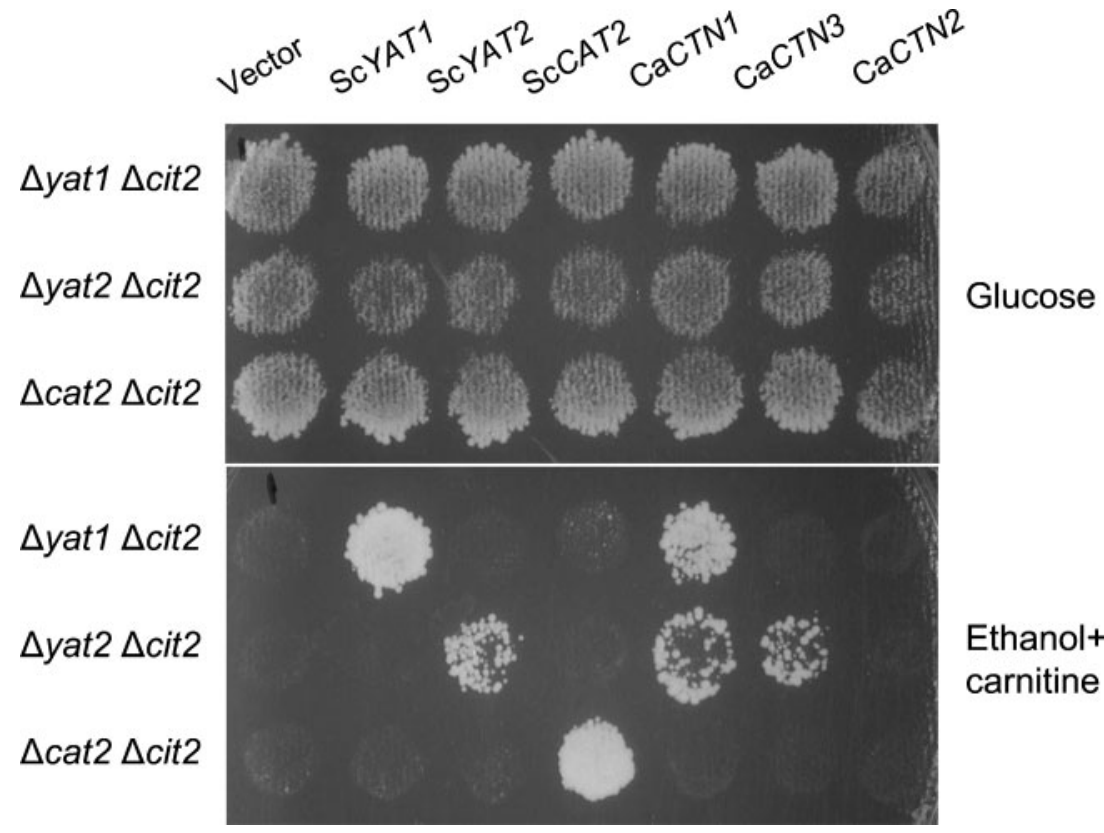

Fig. 4. Complementation of the $S$. cerevisiae mutants. S. cerevisiae strains $\Delta$ yat1 $\Delta$ cit2, $\Delta$ yat2 $\Delta$ cit2 and $\Delta$ cat2 $\Delta$ cit2 were transformed with pRS426-GPD-ScYAT1, ScYAT2, ScCAT2, CaCTN1, CaCTN3 and CaCTN2 respectively. Transformants were patched to minimal YNB medium containing $2 \%$ glucose and incubated at $30{ }^{\circ} \mathrm{C}$ for 3 days before replica plating to minimal YNB medium containing $2 \%$ glucose, or $2 \%$ ethanol plus carnitine $\left(10 \mathrm{mg} \mathrm{l}^{-1}\right)$, and incubated at $30^{\circ} \mathrm{C}$ for 3 (glucose) or 10 (ethanol) days.

peroxisome and the mitochondrial matrix while Ctn1p is associated with the mitochondrial outer membrane (see Fig. 1). Combined with these localizations, our data suggest that acetyl-CoA is converted to acetylcarnitine on the mitochondrial surface by Ctn1p, transported into the matrix, and converted back to acetyl-CoA by Ctn2p before incorporation into the TCA cycle. During growth on fatty acids, the acetyl-CoA produced by peroxisomal $\beta$-oxidation is converted to acetylcarnitine by Ctn2p, and could move from the peroxisome to the mitochondria (via the cytosol) with no need for Ctn1p and, indeed, $\Delta c_{\text {tn } 1}$ mutants can use oleate as a carbon source. The weak growth of $\Delta c t n 2$ mutants on citrate is potentially explained by the presence of a mitochondrial citrate transport protein; C. albicans has an uncharacterized homologue of the well-studied S. cerevisiae Ctn1p protein (Kaplan, et al., 1996), thus citrate can probably enter the mitochondria in the absence of CATs. The phenotypes we observed clearly indicate a need for CAT activity during citrate metabolism, however.
As previously reported, $\Delta c t n 3$ strains do not have carbon utilization phenotypes that we could detect (Prigneau et al., 2004). CTN1 complemented deletions in both S. cerevisiae YAT1 and YAT2, while CTN3 complemented only the syat 2 mutation. This demonstrates a basic conservation of function of these proteins, though this is not absolute, since none of these could restore growth to a $S$. cerevisiae $\Delta$ cat2 mutant.

In an earlier study, CTN3 was found to affect filamentous growth, as $\Delta c t n 3$ strains were unable to form hyphae in several inducing conditions (Prigneau et al., 2004). In contrast, we report here that deletion of the CTN genes did not disrupt formation of hyphae. While there may be some differences in strain background, both studies used SC5314-derived strains. In the study of Prigneau et al. (2004), the CTN3 mutant strains were constructed using the 'URA blaster' method (Fonzi \& Irwin, 1993). This discrepancy may be related to URA3 'position effects', which have been subsequently recognized to cause
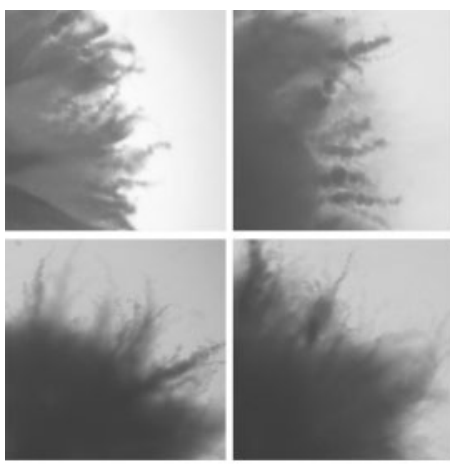

$\operatorname{SC5} 314$
$\Delta c t n 1$

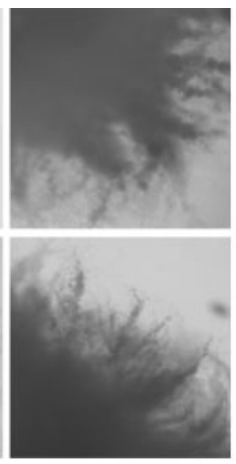

$\Delta c \operatorname{tn} 3$

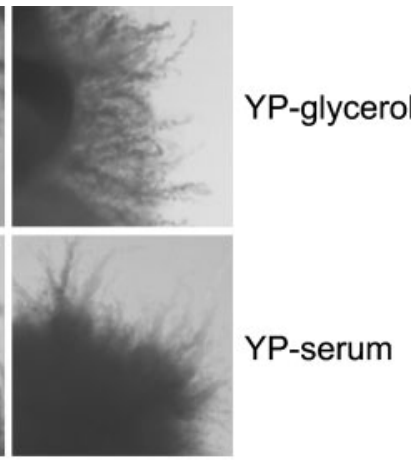

$\Delta c t n 2$
Fig. 5. Peripheral filaments of C. albicans. wild-type (SC5314), $\Delta c t n 1$ (MLC48), $\Delta c t n 3$ (MLC50) and $\Delta c t n 2(\mathrm{HZC} 30)$ strains from fresh YPD plates were struck to single colonies on YP medium containing $3 \%$ glycerol or $10 \%$ serum. Plates were incubated at $37{ }^{\circ} \mathrm{C}$ for 4 days before photographing under a Zeiss dissecting scope. 

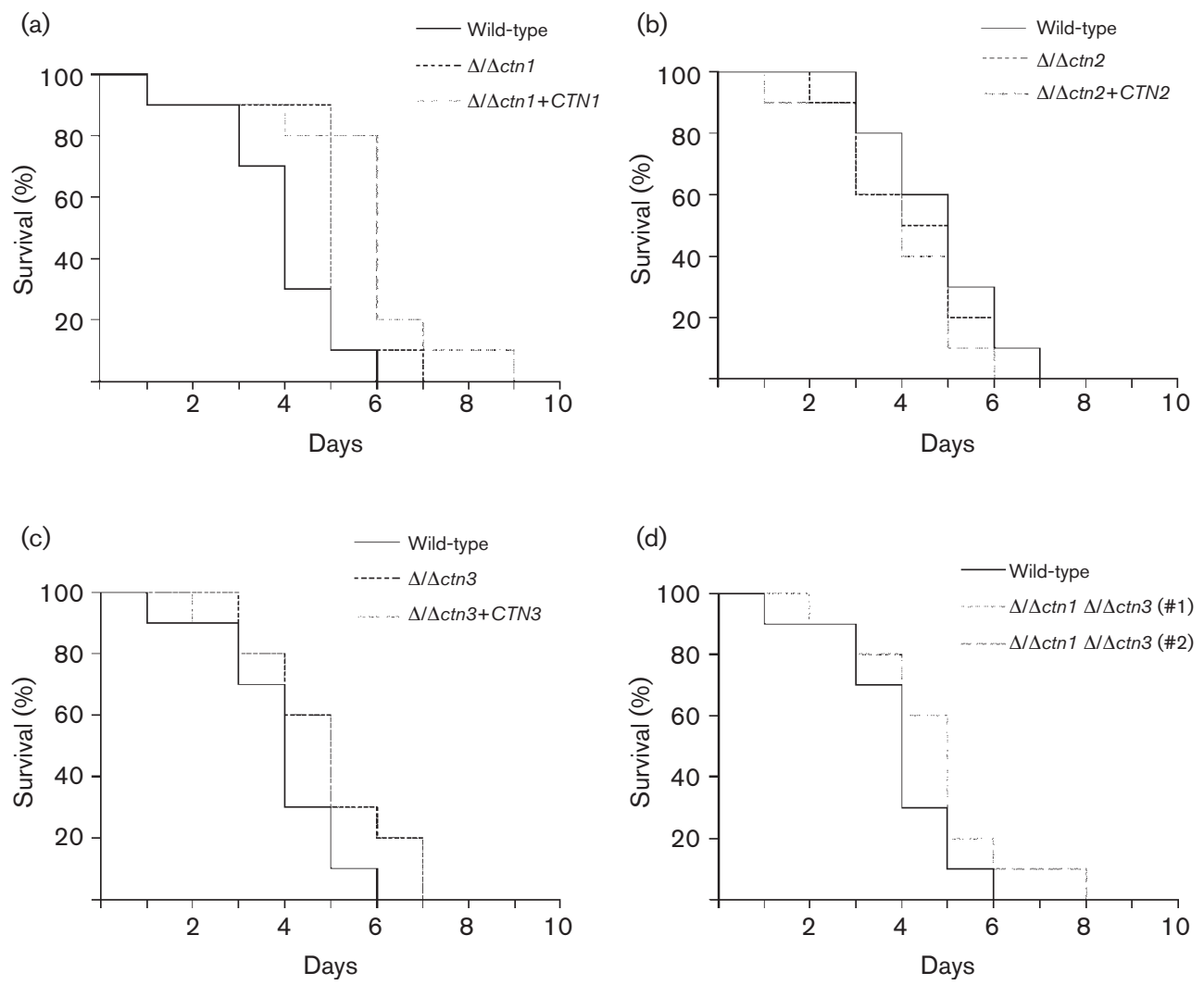

Fig. 6. Virulence test in the mouse model of disseminated candidiasis. A total of $10^{6}$ cells of each strain were injected via the tail vein. All assays used the wild-type strain SC5314 as a control. (a) $\Delta c \operatorname{ctn} 1$ (MLC48) vs $\Delta c t n 1:$ : CTN1 (MLC58); (b) $\Delta c t n 2$ (HZC30) vs $\Delta c t n 2:: C T N 2$ (HZC32); (c) $\Delta c t n 3$ (MLC50) vs $\Delta c t n 3:: C T N 3$ (MLC60); (d) $\Delta c t n 1 \Delta c t n 3$ double mutant (MLC53).

numerous phenotypes, including morphogenetic, that are unrelated to the deleted gene (Bain et al., 2001; Brand et al., 2004; Lay et al., 1998). The current study did not use ura3 auxotrophic strains; instead the mutations were constructed in a prototrophic strain using a dominant drugresistance marker, nourseothricin (Reuß et al., 2004).

Although the CTN mutant strains had dramatic growth defect on non-fermentable carbon sources in vitro, there was no attenuation of virulence in the systemic infection model. We found this result surprising, given that the in vitro phenotypes of the $\Delta c t n$ strains are similar to those of published mutants with known virulence defects, such as $\Delta i c l 1, \Delta f b p 1, \Delta p c k 1$ and $\Delta$ fox2 (Lorenz \& Fink, 2001; Piekarska et al., 2006; Ramírez \& Lorenz, 2007; Barelle et al., 2006). This discrepancy may result from greater expression of the CTN genes in the host environment, mitigating effects of single gene deletions on overall CAT activity. Alternatively, $C$. albicans could import simple carbon molecules, such as acetate or lactate, from the host that do not require intracellular transport for utilization or that make use of other transport systems, such as the mitochondrial succinate-fumarate shuttle or the citrate transporter. In a co-culture assay with murine macrophage cell line J774A, all the deletion strains were able to form hyphae and escape from macrophage engulfment at the same rates as the wild-type control (data not shown). This has been observed previously, even in strains with in vivo virulence defects (Lorenz \& Fink, 2001; Ramírez \& Lorenz, 2007) and we believe that the short duration of the macrophage assay does not allow sufficient time to reveal starvation-related defects.

In contrast to the $\Delta c t n 1$ and $\Delta c t n 2$ mutations, there is no phenotype associated with the deletion of the $S$. cerevisiae CAT genes (YAT1, YAT2, CAT2) (Kispal et al., 1993; Schmalix \& Bandlow, 1993; Swiegers et al., 2001). Indeed, any of these single mutants will grow on non-fermentable carbon sources unless the CIT2 gene, encoding a peroxisomal isoform of citrate synthase, is also deleted. This indicates a flexibility not present in C. albicans, an idea reinforced by the presence of three CIT isoforms in $S$. cerevisiae, and only a single gene in C. albicans (CIT1, highly homologous to all three $S$. cerevisiae proteins). This redundancy likely allows $S$. cerevisiae to run much of the glyoxylate cycle in either the peroxisome or mitochondria and increases the spectrum of compounds that could be shuttled between compartments, thereby reducing the 
importance of acetyl-CoA transport under most circumstances. Why this is the case is not clear, but it is tempting to speculate that it improves the efficiency of ethanol utilization, a process more important in the highly fermentative S. cerevisiae than in other yeasts.

Though these carbon metabolic pathways and enzymes are highly conserved, there have been several previous examples in which gene deletions do not have the same phenotype in these related species. Snflp, a kinase involved in glucose repression, is essential in C. albicans, but not in S. cerevisiae (Petter et al., 1997). In addition, mutations of the C. albicans fox 2 gene, encoding an enzyme of $\beta$ oxidation, has several pleiotropic phenotypes not seen in the S. cerevisiae mutant (Piekarska et al., 2006; Ramírez \& Lorenz, 2007). It is worth considering whether the more restricted localization of carbon metabolic pathways in $C$. albicans that we propose here underlies this pleiotropy. The adaptation of metabolic networks to suit the in vivo niche of C. albicans is likely to be an important aspect of the biology of this pathogen.

\section{ACKNOWLEDGEMENTS}

We thank A. Carman and M. Ramírez for assistance with the mouse virulence assays and A. Carman and K. Morano for comments on the manuscript. This work was supported in part by NIH awards 1R01AI075091-01 and 5T32DE015355-05.

\section{REFERENCES}

Atomi, H., Ueda, M., Suzuki, J., Kamada, Y. \& Tanaka, A. (1993). Presence of carnitine acetyltransferase in peroxisomes and in mitochondria of oleic acid-grown Saccharomyces cerevisiae. FEMS Microbiol Lett 112, 31-34.

Ausubel, F. M., Brent, R., Kingston, R. E., Moore, D. D., Seidman, J. G., Smith, J. A. \& Struhl, K. (editors) (1993). Current Protocols in Molecular Biology. New York: Greene and Wiley-Interscience.

Bain, J. M., Stubberfield, C. \& Gow, N. A. R. (2001). Ura-statusdependent adhesion of Candida albicans mutants. FEMS Microbiol Lett 204, 323-328.

Barelle, C. J., Priest, C. L., MacCallum, D. M., Gow, N. A. R., Odds, F. C. \& Brown, A. J. P. (2006). Niche-specific regulation of central metabolic pathways in a fungal pathogen. Cell Microbiol 8, 961-971.

Brachmann, C. B., Davies, A., Cost, G. J., Caputo, E., Li, J., Hieter, P. \& Boeke, J. D. (1998). Designer deletion strains derived from Saccharomyces cerevisiae S288C: a useful set of strains and plasmids for PCR-mediated gene disruption and other applications. Yeast 14, 115-132.

Brand, A., MacCallum, D. M., Brown, A. J. P., Gow, N. A. R. \& Odds, F. C. (2004). Ectopic expression of URA3 can influence the virulence phenotypes and proteome of Candida albicans but can be overcome by targeted reintegration of URA3 at the RPS10 locus. Eukaryot Cell 3, 900-909.

Elgersma, Y., van Roermund, C. W., Wanders, R. J. \& Tabak, H. F. (1995). Peroxisomal and mitochondrial carnitine acetyltransferases of Saccharomyces cerevisiae are encoded by a single gene. EMBO J 14, 3472-3479.

Fonzi, W. A. \& Irwin, M. Y. (1993). Isogenic strain construction and gene mapping in Candida albicans. Genetics 134, 717-728.
Fradin, C., Kretschmar, M., Nichterlein, T., Gaillardin, C., d'Enfert, C. \& Hube, B. (2003). Stage-specific gene expression of Candida albicans in human blood. Mol Microbiol 47, 1523-1543.

Goldstein, A. L. \& McCusker, J. H. (1999). Three new dominant drug resistance cassettes for gene disruption in Saccharomyces cerevisiae. Yeast 15, 1541-1553.

Kaplan, R. S., Mayor, J. A., Kakhniashvili, D., Gremse, D. A., Wood, D. O. \& Nelson, D. R. (1996). Deletion of the nuclear gene encoding the mitochondrial citrate transport protein from Saccharomyces cerevisiae. Biochem Biophys Res Commun 226, 657-662.

Kispal, G., Sumegi, B., Dietmeier, K., Bock, I., Gajdos, G., Tomcsanyi, T. \& Sandor, A. (1993). Cloning and sequencing of a cDNA encoding Saccharomyces cerevisiae carnitine acetyltransferase. Use of the cDNA in gene disruption studies. J Biol Chem 268, 1824-1829.

Lay, J., Henry, L. K., Clifford, J., Koltin, Y., Bulawa, C. E. \& Becker, J. M. (1998). Altered expression of selectable marker URA3 in genedisrupted Candida albicans strains complicates interpretation of virulence studies. Infect Immun 66, 5301-5306.

Lorenz, M. C. \& Fink, G. R. (2001). The glyoxylate cycle is required for fungal virulence. Nature 412, 83-86.

Lorenz, M. C., Bender, J. A. \& Fink, G. R. (2004). Transcriptional response of Candida albicans upon internalization by macrophages. Eukaryot Cell 3, 1076-1087.

Mumberg, D., Muller, R. \& Funk, M. (1995). Yeast vectors for the controlled expression of heterologous proteins in different genetic backgrounds. Gene 156, 119-122.

Petter, R., Chang, Y. C. \& Kwon-Chung, K. J. (1997). A gene homologous to Saccharomyces cerevisiae SNF1 appears to be essential for the viability of Candida albicans. Infect Immun 65, 4909-4917.

Piekarska, K., Mol, E., van den Berg, M., Hardy, G., van den Burg, J., van Roermund, C., MacCallum, D., Odds, F. \& Distel, B. (2006). Peroxisomal fatty acid $\beta$-oxidation is not essential for virulence of Candida albicans. Eukaryot Cell 5, 1847-1856.

Prigneau, O., Porta, A. \& Maresca, B. (2004). Candida albicans CTN gene family is induced during macrophage infection: homology, disruption and phenotypic analysis of CTN3 gene. Fungal Genet Biol 41, 783-793.

Ramírez, M. A. \& Lorenz, M. C. (2007). Mutations in alternative carbon utilization pathways in Candida albicans attenuate virulence and confer pleiotropic phenotypes. Eukaryot Cell 6, 280-290.

Reuß, O., Vik, A., Kolter, R. \& Morschhauser, J. (2004). The SAT1 flipper, an optimized tool for gene disruption in Candida albicans. Gene 341, 119-127.

Rubin-Bejerano, I., Fraser, I., Grisafi, P. \& Fink, G. R. (2003). Phagocytosis by neutrophils induces an amino acid deprivation response in Saccharomyces cerevisiae and Candida albicans. Proc Natl Acad Sci U S A 100, 11007-11012.

Schmalix, W. \& Bandlow, W. (1993). The ethanol-inducible YAT1 gene from yeast encodes a presumptive mitochondrial outer carnitine acetyltransferase. J Biol Chem 268, 27428-27439.

Sexton, J. A., Brown, V. \& Johnston, M. (2007). Regulation of sugar transport and metabolism by the Candida albicans Rgt1 transcriptional repressor. Yeast 24, 847-860.

Swiegers, J. H., Dippenaar, N., Pretorius, I. S. \& Bauer, F. F. (2001). Carnitine-dependent metabolic activities in Saccharomyces cerevisiae: three carnitine acetyltransferases are essential in a carnitinedependent strain. Yeast 18, 585-595.

van Roermund, C. W., Elgersma, Y., Singh, N., Wanders, R. J. \& Tabak, H. F. (1995). The membrane of peroxisomes in Saccharomyces 
cerevisiae is impermeable to $\mathrm{NAD}(\mathrm{H})$ and acetyl-CoA under in vivo conditions. EMBO J 14, 3480-3486.

Wach, A., Brachat, A., Pohlmann, R. \& Philippsen, P. (1994). New heterologous modules for classical or PCR-based gene disruptions in Saccharomyces cerevisiae. Yeast 10, 1793-1808.

Wenzel, R. P. (1995). Nosocomial candidemia: risk factors and attributable mortality. Clin Infect Dis 20, 1531-1534.

Winzeler, E. A., Shoemaker, D. D., Astromoff, A., Liang, H., Anderson, K., Andre, B., Bangham, R., Benito, R., Boeke, J. D. \& other authors (1999). Functional characterization of the $S$. cerevisiae genome by gene deletion and parallel analysis. Science 285, 901-906.

Wisplinghoff, H., Bischoff, T., Tallent, S. M., Seifert, H., Wenzel, R. P. \& Edmond, M. B. (2004). Nosocomial bloodstream infections in US hospitals: analysis of 24,179 cases from a prospective nationwide surveillance study. Clin Infect Dis 39, 309-317.

Edited by: J. M. Becker 